In conclusion, $\mathrm{Xu}$ and colleagues' fantastic work solves a controversial issue and provides a proof of principle for similar studies in other solid tumours. Once again, retinoblastoma acts as a model for the cancer field. Knowledge of the cell of origin for retinoblastoma (and other cancers) may help researchers to develop approaches for better diagnosis, earlier detection, and possibly chemoprevention. In addition, a better understanding of the molecular circuitry that renders cells susceptible to cancerous transformation may help to uncover Achilles heels in tumour cells. -

Rod Bremner is at Mount Sinai Hospital, Lunenfeld Tanenbaum Research Institute, Toronto, Ontario M5G 1X5, Canada. Julien Sage is in the Departments of Pediatrics and Genetics, Stanford University, Stanford, California 94305, USA. e-mails:bremner@lunenfeld.ca; julsage@stanford.edu

1. Xu, X. L. et al. Nature 514, 385-388 (2014)

2. Friend, S. H. et al. Nature 323, 643-646 (1986).

3. Visvader, J. E. Nature 469, 314-322 (2011).

4. Sage, J. Genes Dev. 26, 1409-1420 (2012).

5. Kyritsis, A. P., Tsokos, M., Triche, T. J. \& Chader, G. J. Nature 307, 471-473 (1984)

6. Rootman, D. B. et al. Br. J. Ophthalmol. 97, 59-65 (2013).

7. Calo, E. et al. Nature 466, 1110-1114 (2010).

8. Dyer, M. A. \& Bremner, R. Nature Rev. Cancer 5, 91-101 (2005).

9. Sangwan, M. et al. Oncogene 31, 5019-5028 (2012).

10.McEvoy, J. et al. Cancer Cell 20, 260-275 (2011).

11.Conkrite, K., Sundby, M., Mu, D., Mukai, S. \& Macpherson, D. J. Clin. Invest. 122, 1726-1733 (2012).

12.Xu, X. L. et al. Cell 137, 1018-1031 (2009).

13.Chen, D., Chen, Y., Forrest, D. \& Bremner, R. Cell Death Differ. 20, 931-940 (2013).

This article was published online on 24 September 2014.

\title{
A historic experiment redesigned
}

Large quasiparticles known as Rydberg excitons have been detected in a natural crystal of copper oxide. The result may find use in applications such as single-photon logic devices. SEE LETTER P.343

\section{SVEN HÖFLING \& ALEXEY KAVOKIN}

A $\mathrm{n}$ exciton is a quasiparticle in a solidstate system comprising an electron and a hole (the absence of an electron). It has an energy spectrum akin to that of a hydrogen atom, and so may be considered as an artificial hydrogen atom in a solid-state environment, with the hole playing the part of the hydrogen's proton. The concept of excitons was first formulated in the early 1930s by Yakov Frenkel ${ }^{1}$, who predicted their existence in molecular crystals. A few years later, Gregory Wannier ${ }^{2}$ and Nevill Mott $^{3}$ described these electron-hole bound states for inorganic semiconductors. In 1952, Evgeniy Gross and Nury Karryjew ${ }^{4}$ discovered these Wannier-Mott excitons experimentally in a copper oxide $\left(\mathrm{Cu}_{2} \mathrm{O}\right)$ semiconductor. Now, on page 343 of this issue, Kazimierczuk et al. ${ }^{5}$ report how they have redesigned this historic experiment to find excitons in a natural crystal of copper oxide. The excitons extend across some tens of billions of lattice sites of the crystal.

Gross and Karryjew's discovery marked the beginning of 'excitonics' - an area of solidstate physics that holds promise for applications in optoelectronics and in information and communication technologies ${ }^{6}$. For their studies, Gross and Karryjew selected crystals of copper oxide, and, using a spectrograph, identified eight dark lines in the material's transmission spectrum. Such absorption dips indicated the energies of optically induced transitions from the crystal's ground state to excited states with principal quantum numbers $n=2,3, \ldots 9$ (Fig. 1). The transition energies scaled with $n$ in a similar way to those of a hydrogen atom. This result proved that hydrogen-like quasiparticles, excitons, can be generated in these semiconductor crystals by photoabsorption.

In their study, Kazimierczuk et al. performed high-resolution transmission spectroscopy of an extremely high-quality natural crystal of copper oxide found at the Tsumeb mine in Namibia using laser light of tunable frequency and ultralow spectral linewidth (corresponding to roughly 1.2 megahertz). Taking advantage of the narrow linewidth of the laser and the high purity of the crystal, the authors have measured transmission spectra of the material with a spectral resolution of 5 nanoelectronvolts - an extremely high value for optical spectroscopy experiments. Analysis of the spectra revealed absorption lines associated with excitons with principal quantum numbers as large as $n=25$. The size of an exciton increases as $n^{2}$, with $n=25$ corresponding to a

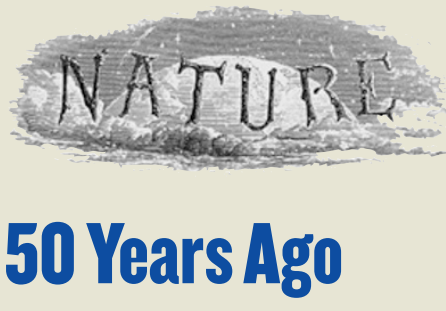

In general, the 'epidemic' process can be characterized as one of transition from one state (susceptible) to another (infective) where the transition is caused by exposure to some phenomenon (infectious material) ... People are susceptible to certain ideas and resistant to others. Once an individual is infected with an idea he may in turn, after some period of time, transmit it to others. Such a process can result in an intellectual 'epidemic' ... The development of the psychoanalytic movement in the early part of the twentieth century was in its way no less an 'epidemic' than was the outbreak of influenza in 1917 and 1918. One can argue similarly that Darwin and evolution, Cantor and set theory, Newton and mechanics, and so on, were examples of 'epidemics' in the world of scientific thought which were instigated by the introduction of a single infective into a population. From Nature 17 October 1964

100 Years Ago

At the present time astronomers have no available organisation by which the news of important astronomical discoveries can be quickly distributed to the leading observatories of the world, nor is there a bureau with which anyone making an important discovery can immediately communicate with the knowledge that the news will at once be circulated world wide. This condition of affairs is due to the fact that the recognised Central Bureau is at Kiel, in Germany, and that the state of war prevents the circulation of any such news ... There is little doubt that if the Royal Astronomical Society of Great Britain would undertake ... the task of receiving and disseminating astronomical information, this act would meet with the approval of astronomers all the world over. From Nature 15 October 1914 